\title{
KNOWLEDGE ACQUISITION AND REPRESENTATION OF THE GENERAL AUXILIARY DIAGNOSIS SYSTEM FOR COMMON DISEASE OF ANIMAL
}

\author{
Jianhua Xiao, Hongbin Wang*, Ru Zhang, Peixian Luan, Lin Li, Danning \\ $\mathrm{Xu}$ \\ College of Veterinary Medicine, NorthEast Agricultural University, Harbin,Heilongjiang \\ Province, P. R. China, 150030 \\ * Corresponding author, Address: College of Veterinary Medicine, NorthEast Agricultural \\ University, Harbin,150030, Heilongjiang Province, P. R. China, Tel: +86-451-55191940, \\ Fax:+86-451-55190470,Email: neau1940@yahoo.com.cn
}

Abstract: The main methods of diagnosis for animal is symptom, generally the end diagnosis conclusion can be get by experiment after diagnosis by symptoms, for this reason, the symptom, epidemic character and pathological changes are most important factors in diagnosis. Based on the level of diagnosis in china, the knowledge acquisition and representation methods were stated in this papers, the animal disease diagnosis knowledge was acquired from experts in animal disease and books, the knowledge was represented in rule, and one factor is assigned to each condition of a rule, representing its significance in drawing the conclusion. This results in better representation, and facilitates knowledge acquisition and maintenance.

Key words: diagnosis, knowledge acquisition, knowledge representation

\section{INTRODUCTION}

Computer-based methods are increasingly used to improve the efficacy and effects of veterinary medical services. In medical diagnosis, expert system is mainly used for performing diagnoses based on symptom of patient, since they can naturally represent the way expert's reason. Diagnosis

Please use the following format when citing this chapter:

Xiao, J., Wang, H., Zhang, R., Luan, P., Li, L. and Xu, D., 2009, in IFIP International Federation for Information Processing, Volume 294, Computer and Computing Technologies in Agriculture II, Volume 2, eds. D. Li, Z. Chunjiang, (Boston: Springer), pp. 947-952. 
of animal diseases is greatly facilitated by the symptoms and epidemic characters but not by experiment or pathological change just as pig, chicken etc. In this paper, knowledge acquisition and representation of the general auxiliary diagnosis system for common disease of animal was presented.

\section{KNOWLEDGE ACQUISITION}

Generally, the data of patient animal can be distinguished in three types: epidemic symptoms, pathological changes and laboratory results. Symptoms are those detected by a physical examination of the patient, like e.g. the existence and the kind of a pain etc. pathological changes are those detected by ptomatopsia and check by microscope. Laboratory results are those detected via laboratory tests, like e.g. blood tests etc. the knowledge for diagnosis disease is the inference from patient data to disease. In another words, what disease the animal get if it has one symptom. This kind of knowledge alike declarative knowledge very much, but not belong to it. Declarative knowledge refers to knowing that something is true or false. It is concerned with knowledge expressed in the form of declarative statements such as "sky is blue". But the diagnosis knowledge for disease can not be affirmed directly (Joseph C, 2005). This type of knowledge is fuzzy and represented by Fuzzy technique better than traditional two-valued logic. For this reason, the diagnosis knowledge for animal diseases should be obtained from expert by analysis but not by statistics. The knowledge in this system obtained from expert and books about animal disease. The knowledge from book was arranged as the follow form by some postgraduates at begins:

If there is one symptom alike "lameness" then there is cf possibility

That the animals get foot-and-mouth disease

Then this statement was filled into one table as below:

Table.1 the form of knowledge when Acquisition

\begin{tabular}{lll}
\hline Patient data & disease & cf \\
\hline lameness & foot-and-mouth & \\
Cough & pneumonia & \\
Frequent micturition & nephritis & \\
\multicolumn{1}{c}{$\ldots$} & & $\ldots$ \\
\hline
\end{tabular}

And then the confidence of this knowledge was determined by expert. In fact, it is very difficult to analyze the possibility of this symptom result in this disease. And it is not useful if the knowledge were obtained in this rambling form. Because one symptom may signify many diseases, and one disease may show many symptoms. Therefore, if the confidence of 
knowledge be analyzed leave other knowledge alone, the confidence of this knowledge would separate with that knowledge which has close relation with symptom or disease in this knowledge. The relations among different knowledge (see figure.1) were disregarded consequently.

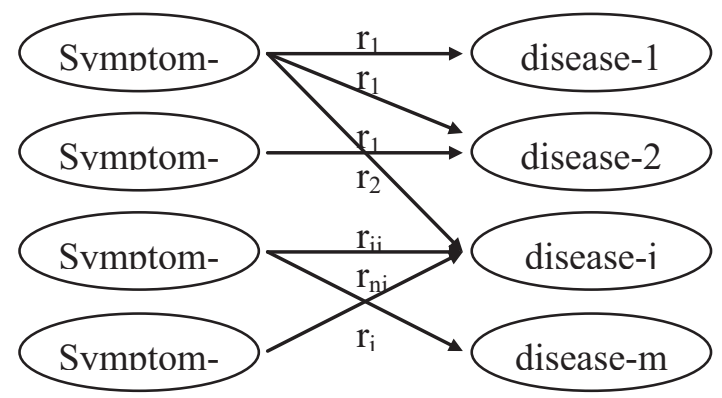

Figure.1 the inner relation among different knowledge for diagnosing of animal disease

$\mathrm{r}_{\mathrm{ij}}$ represent the rule between symptom- $i$ and disease- $j$. if symptom-1 appeared in one animal then disease-1, disease-2, disease-j can be occurred. On the contrary, if disease-I occurred then symptom-1, symptom-i and symptom-n can be appeared. Therefore when the confidence of $r_{11}$ will be analyzed, the confidence of $r_{12}, r_{1 n}, r_{i j}, r_{n j}$ should be analyze in same time. Although one disease may show many symptoms, but these symptoms can be divided into two types: common symptom and typical symptom.

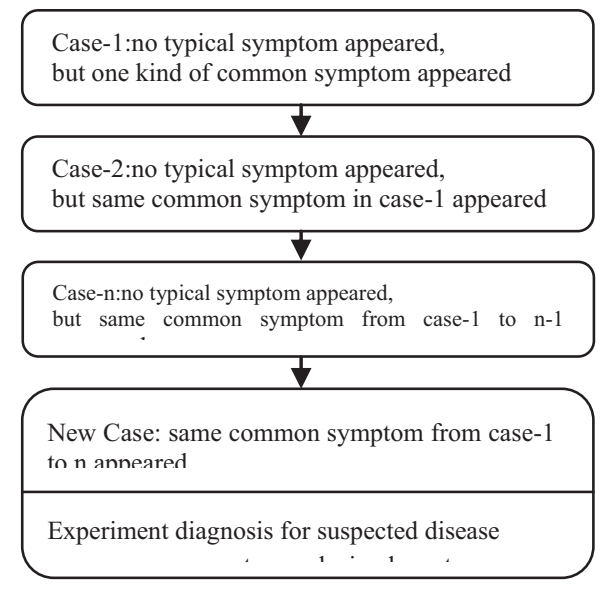

Diaqnosis by common symptom by

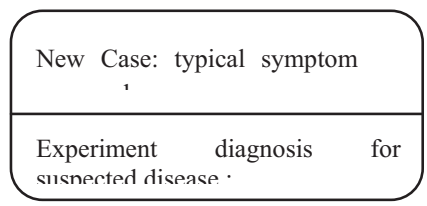

Diagnosis by typical

Fig.2 The different diagnosis ways for by typical symptom and common symptom

Generally, there are 4 kinds of results may occur when diagnose by inexperienced doctor and expert in practice: 
Situation 1: the typical symptom appears, the expert make most accurate diagnosis at once

Situation 2: the typical symptom appears, the inexperienced doctor relatively accurate diagnosis

Situation 3: only common symptoms appears, the expert make relatively accurate diagnosis by his experience

Situation 4: only common symptoms appears, the inexperience doctor make inaccurate diagnosis by his experience

Situation 1 is the most ideal result in practice, however true expert with plenty of experience and theory are scarce. Moreover, typical symptom seldom appeared in practice. Therefore, one relatively accurate diagnosis can be made by one doctor with many years' experience usually. By above discussion, one conclusion can be reached:

The accurate of diagnosis was determined on two factors: typical symptom and experience. If the typical symptom do not appeared, the expert can make one relatively accurate by his many experiences on same case (see fig.2).

\section{KNOWLEDGE REPRESENTATION}

Representing medical knowledge is a highly complex endeavor. The improper use of the terms "syndrome", "disease" and their relations to "diagnosis" is one of the difficulties with which medical informaticians must deal, especially when developing expert systems to support diagnoses(). A situation met in medical diagnosis is the following. There are a number of symptoms that all contribute in diagnosing a disease. However, not all of the symptoms have the same significance. For example, a symptom S1 may give much stronger evidence for diagnosing a disease D than a symptom S2. Also, some combinations of symptoms may give stronger evidence than others in diagnosing the disease. To be able to represent this situation in production rules formalism, we introduce a factor assigned to each condition of a rule, representing its significance in drawing the conclusion. The knowledge was represented as the follow form:

\section{If condition then conclusion cf}

Because the count of knowledge may be very great, the inference may be very difficult. Otherwise, different knowledge plays a different role in inference. The knowledge based on typical symptoms play a determined role in diagnosis, but common symptom play a not important role. If one typical symptom appears then there is a great possibility that one conclusion can be make, while some symptom appears can not make any conclusion, in another 
words, these symptom have a little relation with one disease, for example: anorexia. Any disease may result in the decrease of appetite. Therefore the symptoms as anorexia have no help to diagnosis generally. However, anorexia may be very important to diagnosis diseases of digestive system, so one symptom has different role for different diseases. Based on above ideas, a five-grade score was used in this expert system. They are certain not, little possible, possible, very possible, certain. The significance factor $s f$ takes value from these five grades. The principle of take value for one knowledge is list in table.2.

Tab.2 the principle of take value for one knowledge

\begin{tabular}{lll}
\hline score & means & The principle of take value \\
\hline 0 & Certain not & The disease have no relation with this symptom certainly \\
\cline { 2 - 3 } 1-4 & Little possible & $\begin{array}{l}\text { The disease have little relation with this symptom, but this } \\
\text { symptom does not signify this disease }\end{array}$ \\
\cline { 2 - 3 } 5 & Possible & $\begin{array}{l}\text { The disease have relation with this symptom, if this } \\
\text { symptom appears then it is likely that this disease has } \\
\text { occurred, but not very certain }\end{array}$ \\
\cline { 2 - 3 } 6-7 & Very possible & $\begin{array}{l}\text { If this symptom appears then the possibility for this disease } \\
\text { occurred is very great }\end{array}$ \\
\cline { 2 - 3 } $8-10$ & certain & $\begin{array}{l}\text { If this symptom appears then this disease occurred on the } \\
\text { whole }\end{array}$ \\
\hline
\end{tabular}

The above principles reflect the relation between symptom and disease to a great extent. But when acquire knowledge in above principles, a lot of disease may corresponding with one symptom, and one disease may corresponding many symptom. The result may be when one symptom be input a lot of disease may output. Moreover, the system runs slowly. In fact, the essence of disease was not embodied in above principles. Generally diseases of animal can be divided into two categories: infective diseases and noninfective diseases. the symptoms of infective diseases appear in many system, and the symptoms of noninfective diseases appear in few system. Moreover, there was no difference among symptoms just as wet cough, dry cough, and painful cough in essence. The difference among those symptoms is degree. Otherwise, when animal cough, it may wheeze, nose running, even lose breath in the same time. And the symptoms as those are only the typical symptoms of infection disease of respiratory system but not others. Furthermore, by symptoms like cough, wheeze etc. concrete disease can not be inferred. For above reasons, a more complex knowledge representation form was design to solve above problem. The new representation form is:

\section{If condition (class) then conclusion cf is-Typical}

The "class" represents the relation among different condition for one disease. Class is take value from "X1, $\mathrm{X} 2, \mathrm{Xn}$; Y1, Y2, Yn; Z1, Z2, Zn", 
$\mathrm{n}<10$. $\mathrm{X} \mathrm{Y}, \mathrm{Z}$ means this condition belong to group $\mathrm{X}$, group $\mathrm{Y}$ and group $\mathrm{Z}$ respectively. $1,2, \cdots, \mathrm{n}$ means any class which include $1,2, \mathrm{n}$, be selected the sum weight will be add 1, 2, , n respectively. "is-Typical" take value from "is" or "not", if "is" then means the condition in this knowledge is a typical symptom. Therefore by this condition one conclusion can be making certainly.

\section{CONCLUSION}

The accurate was relative low in veterinary medical. There was more problem not solved in veterinary medical than human medical. Therefore one human doctor will get much knowledge than one veterinary doctor with same time and energy. Maybe the veterinary medicine has developed to the same level as human medicine in some area. But the level was relative low in most area. There are more than 100 kinds of animal disease occurred usually. These diseases belong to 8 systems. Every disease will show several symptoms. Therefore, it is a more complex procedure of diagnosis for animal disease than disease of human being. The knowledge representation methods in rule are one simple but effective ways.

\section{ACKNOWLEDGEMENTS}

Thanks to surgical department faculty working office of the Northeast Agricultural universdity, as well as Professor Liu Yun, associate professor Gao Li by the help regarding to this research.

\section{REFERENCES}

C Joseph, Giarratano, D Gary. Riley. Expert Systems Principles and Programming, 4th, Edition. Thomson Learning Ltd, 2005.

S.V. Ellam, M.N. Maisen. A Knowledge-based System to Assist in the Diagnosis of Thyroid Disease from a Radioisotope Scan, in Pretschner D.P. and Urrutia B., (Eds), Knowledgebased systems to aid medical image analysis, vol.1, Commission of the European Community, 1990. 